\title{
Administration of erythropoietin prevents bone loss in osteonecrosis of the femoral head in mice
}

\author{
TAOTAO XU ${ }^{1-3^{*}}$, HONGTING JIN ${ }^{1,2^{*}}$, YANGJUN LAO ${ }^{1,2}$, PINGER WANG $^{1,2}$, SHANXING ZHANG $^{4}$, \\ HONGFENG RUAN $^{1,2}$, QIANG MAO ${ }^{3}$, LI ZHOU ${ }^{1,2}$, LUWEI XIAO ${ }^{4}$, PEIJIAN TONG ${ }^{4}$ and CHENGLIANG WU ${ }^{1,2}$ \\ ${ }^{1}$ Zhejiang Chinese Medical University; ${ }^{2}$ Institute of Orthopaedics and Traumatology of \\ Zhejiang, Hangzhou, Zhejiang 310053; ${ }^{3}$ Department of Orthopaedic Surgery, School of Medicine, \\ Washington University, St. Louis, MO 63110, USA; ${ }^{4}$ Department of Orthopaedic Surgery, The First \\ Affiliated Hospital of Zhejiang Chinese Medical University, Hangzhou, Zhejiang 310006, P.R. China
}

Received April 6, 2017; Accepted August 22, 2017

DOI: $10.3892 / \mathrm{mmr} .2017 .7735$

\begin{abstract}
Long-term administration of glucocorticoid hormones is considered one of predominant pathological factors inducing osteonecrosis of the femoral head (ONFH) development and progression, in which reduction of blood supply leads to a progressive bone loss and impairment of bone structure in the majority of cases. In a non-hematopoietic system, erythropoietin (EPO) can stimulate angiogenesis and bone regeneration. However, the specific mechanism underlying the role of EPO in ONFH remains to be elucidated. Therefore, the purpose of this study was to determine the effect of EPO on the prevention of bone loss in ONFH. Male C57BL/6J mice 3 months old were divided into two groups: EPO group and control groups. ONFH was established by the administration prednisolone (PDS, $100 \mathrm{mg} / \mathrm{kg}$ ) with co-treatment of lipopolysaccharide (LPS, $1 \mathrm{mg} / \mathrm{kg}$ ). ONFH mice received recombinant mouse EPO (500 U/kg/day) or saline intramuscularly. The mice were sacrificed at 2, 4, 6 and 8 weeks following the initiation of treatment. Alterations in the general architecture and histomorphology of the right femoral head were determined by hematoxylin and eosin staining and micro computed tomography (micro-CT). The expression of runt-related transcription factor 2 (Runx2), osteocalcin, vascular endothelial growth factor (VEGF) and platelet endothelial cell adhesion molecule (CD31) in the femoral head was tested by immunohistochemistry. Terminal deoxynucleotidyl-transferase-mediated dUTP nick end labeling (TUNEL) assay was performed to detect apoptosis in femoral heads.
\end{abstract}

Correspondence to: $\mathrm{Dr}$ Chengliang Wu, Zhejiang Chinese Medical University, 548 Bin Wen Road, Hangzhou, Zhejiang 310053, P.R. China

E-mail: chengliangwuzjtcm@163.com

*Contributed equally

Key words: erythropoietin, bone loss, osteonecrosis of femoral head, osteogenesis, angiogenesis
Micro-CT data revealed that EPO significantly improved bone volume/total volume and bone mineral density following 6 and 8 weeks of treatment. Histological analysis further demonstrated that EPO treatment improved the arrangement of trabeculae, thinning of trabeculae and other fractures in femoral heads, especially following 6 and 8 weeks of treatment. Immunohistochemical analysis suggested that EPO treatment up-regulated the expressions of Runx2, osteocalcin, VEGF and CD31 at 4 and 8 weeks. The TUNEL apoptosis assay suggested that EPO intervention reduced apoptosis in avascular ONFH. Therefore, EPO prevents bone loss in ONFH in mice through enhancing Runx2-mediated osteogenesis, VEGF-mediated angiogenesis and inhibition of cell apoptosis.

\section{Introduction}

Osteonecrosis of the femoral head (ONFH) is a common and progressive disease that predominantly impairs the femoral head (1). With the development of the pathological process, it often progresses and leads to the collapse of the femoral head and osteoarthritis. Late stages of ONFH cause severe pain and require surgical arthrodesis, which severely affects the quality of life and the ability to work, and simultaneously causes economic burden for patients and society. Although multiple factors have been demonstrated to contribute to ONFN, long-term administration of glucocorticoid hormones (GCs) is considered a predominant cause. Currently, surgery is the primary treatment option, which can markedly improve the symptoms among young and middle-age patients. However, multiple revision surgeries are frequently required during the lifetime of the patient. Therefore, there is a need to develop novel medical therapies for the treatment of ONFH.

Erythropoietin (EPO), a pleiotropic cytokine mainly secreted by kidneys, stimulates the production of red blood cells (2). Previous studies reported beneficial effects of EPO on the non-hematopoietic system and on the bone fracture repair process, including stimulation of angiogenesis and bone formation $(3,4)$. Moreover, Bakhshi et al $(5)$ hypothesized that local administration of recombinant human erythropoietin (rhEPO) combined with core decompression surgery can enhance angiogenesis and bone regeneration in the early 
stages of ONFH. However, the mechanism underlying the role of EPO on ONFH remain to be elucidated.

The pathomechanism underlying avascular necrosis of the femoral head induced by steroids can be ultimately ascribed to the damage of the local vasculature and restriction of oxygen supply to the femoral head (6). Vascularization in bone tissue supplies nutrients for the normal metabolic process, and angiogenesis is essential for bone tissue regeneration in the necrotic area of the femoral head. Vascular endothelial growth factor (VEGF) regulates the coupling of angiogenesis and bone formation, and regeneration (7). It has been demonstrated that EPO serves an important role in capillary vessel formation and up-regulation of VEGF mRNA expression during tendon repair (8). In addition, studies have demonstrated that GCs can induce apoptosis of osteoblasts or osteocytes in the steroid-induced ONFH $(9,10)$.

Therefore, the authors of the present study hypothesized that the administration of EPO prevents bone loss in a mouse model of ONFH by increasing osteocalcin- and vascular endothelial growth factor (VEGF)-mediated angiogenesis, up-regulating runt-related transcription factor 2 (Runx2)-mediated osteogenesis and inhibiting cell apoptosis.

\section{Materials and methods}

Animals. In the present study, male C57BL/6J mice (weight, 23.5-29.4 g; age, 3 months; $n=80$ ) were provided by the Center of Experimental Animals, Zhejiang Chinese Medical University (Hangzhou, China). Mice were kept in a temperature- and humidity-controlled environment $\left(22 \pm 1^{\circ} \mathrm{C}, 40-60 \%\right.$ humidity, atmospheric $\mathrm{CO}_{2}$ ) with a free access to standard pellet food and tap water and a 12-h light/dark cycle. The present study was approved by the local Governmental Animal Care Committee (Hangzhou, China) and all the experimental procedures adhered to the guidelines set by the Center of Experimental Animals, Zhejiang Chinese Medical University (Hangzhou, China).

Model of ONFH. The model of ONFH was established to study the effect of EPO treatment on ONFH. Mice were randomly and equally divided into 2 groups: The EPO group and the control group. The mouse model of ONFH was established by the administration of prednisolone (PDS; Reyon Pharmaceutical Co., Ltd., Seoul, Korea) and co-treatment with lipopolysaccharide (LPS; Sigma-Aldrich; Merck KGaA, Darmstadt, Germany), as previously described (11). Intraperitoneal (ip) administration of LPS (1 mg/kg) was immediately followed by an intramuscular injection of PDS (100 mg/kg). A second double-injection was performed 1 week following the initial co-injection of PDS/LPS and injections were continued every 3 days until the mice were sacrificed. The mice in the control group were treated with the same dosing regimen, using saline as a substitute for LPS and PDS.

Intervention. Mice in the EPO group were intramuscularly administered recombinant mouse EPO (500 U/kg/day; cat. no. 959-ME-010; R\&D Systems, Inc., Minneapolis, MN, USA) daily starting 1 week following the initial co-injection of PDS/LPS. The mice in the control group were administered an equivalent amount of saline. At 2, 4, 6 and 8 weeks following intervention, 6 mice from each group were sacrificed by carbon dioxide $\left(\mathrm{CO}_{2}\right)$ narcosis. Subsequently, the mice were dissected and right femurs were harvested. Following removal of the skin and muscle from the legs, the entire femur with the hip joint was removed from the mouse's body by cutting above the hip joint and in the knee joint. Then the hip joint was dissected to expose the femoral head and remove the acetabular tissue. This tissue was used for micro computed tomography (micro-CT) and histological analyses.

Micro-CT analysis. Micro-CT was performed using a Skyscan 1172X-ray microtomograph (resolution, $2.47 \mu \mathrm{m}$; Bruker Corporation, Billerica, MA, USA). Femurs were placed vertically in the microtomograph sample holder and images were captured at $65 \mathrm{kV}(153 \mu \mathrm{A})$ using a $0.5-\mathrm{mm}$ aluminum filter. A series of 483 projection images was captured with a rotation step of $0.40^{\circ}$ between each image for each specimen. For each sample, 3-dimensional projection images were reconstructed from a stack of 2-dimensional images, using the NRecon software (version 1.6.10; Skyscan; Bruker Corporation). In the trabecular region of the femoral head, a $0.8 \times 0.8 \mathrm{~mm}^{3}$ region of interest (ROI) was selected in the center of the femoral head using a semiautomatic contouring method, and the contouring of images was performed every 50 axial slices (proximal to distal). Bone morphometric parameters of ROI, including tissue volume (TV), bone volume (BV) and bone mineral density (BMD), were calculated using CTAn analysis software (version 1.15; Skyscan; Bruker Corporation), as previously described (12).

Histological analysis. Following harvesting of right femoral heads, samples were fixed with $10 \%$ buffered formalin for 3 days and then decalcified in $14 \%$ ethylenediamine tetraacetic acid for 2 weeks. Decalcified samples were embedded in paraffin and $3 \mu \mathrm{m}$ sections were prepared for histological analysis through haematoxylin and eosin (H\&E) staining. The sections were stained in CAT hematoxylin (no. CATHE-MM; Biocare Medical, LLC, Pacheco, CA, USA) for 1 min and counterstained in alcoholic-eosin (no. 17372-87-1; Sigma-Aldrich; Merck KGaA) for $1 \mathrm{~min}$. All the procedures were performed at room temperature. Successful induction of ONFH was defined as diffuse presence of empty lacunae or pyknotic nuclei of bone cells in the trabecular bone, accompanied by surrounding bone marrow cell necrosis $(13,14)$. Histopathological changes of ONFH were examined under a light microscope (Axio Scope A1; Carl Zeiss AG, Oberkochen, Germany) in a blinded manner by three independent investigators. At least 50 lacunae in each field were counted and 5 fields in each slide were selected randomly. The fraction of empty lacunae to total lacunae was defined as the ratio of empty lacuna number to the total lacuna number. The trabecular bone volume in the area of femoral heads was measured by Image-Pro Plus software (version, 6.0; Media Cybernetics, Inc., Rockville, MD, USA) and the percentage of trabecular BV/TV of femoral heads was calculated.

Immunohistochemical analysis. To investigate the expression levels of Runx2, osteocalcin, VEGF and platelet endothelial cell adhesion molecule (CD31) in femoral heads, bone sections were prepared for immunohistochemistry. Following 
deparaffinization in xylene and rehydration in a descending ethanol series (two changes in $100 \%$ ethanol, two changes in $95 \%$ ethanol, one change in $70 \%$ ethanol, and finally in distilled water), endogenous peroxidase was quenched by $3 \%$ hydrogen peroxide $\left(\mathrm{H}_{2} \mathrm{O}_{2}\right)$ for $20 \mathrm{~min}$, and antigen retrieval was performed using $0.01 \mathrm{M}$ citrate buffer $\left[(\mathrm{pH} 6.0), 20 \mathrm{~min}, 95^{\circ} \mathrm{C}\right.$; cat. no. C02-02001; Bioss, Beijing, China]. Following blocking of non-specific binding sites with a normal goat serum (1:20; cat. no. C-0005; Bioss) for $20 \mathrm{~min}$ at room temperature, sections were incubated overnight with mouse monoclonal anti-Runx2 (Cbfa1; 1:100; cat. no. D130-3; MBL International Co., Woburn, MA, USA), rabbit polyclonal anti-osteocalcin $(1 \mu \mathrm{g} / \mathrm{ml}$; cat. no. M173; Takara Biotechnology Co., Ltd., Dalian, China), mouse monoclonal anti-VEGF (VG-1; 1:100; cat. no. ab1316; Abcam, Cambridge, UK) and rabbit polyclonal anti-CD31 (1:50; cat. no. ARG52748; Arigo Biolaboratories, Hsinchu City, Taiwan) primary antibodies. Peroxidase-conjugated goat anti-mouse (1:100; cat. no. HA1006; Hangzhou HuAan Biotechnology Co., Ltd., Hangzhou, China) or peroxidase-conjugated goat anti-rabbit $(1: 1,000$; cat. no. GS1001; Shanghai Good-Science Biotech Company, Shanghai, China) immunoglobulin $\mathrm{G}$ antibodies were used as secondary antibodies, respectively. Diaminobenzidine (DAB) served as a chromogen, and hematoxylin served as a counterstain (20 sec, at room temperature). Tissue sections were examined under a light microscope (Zeiss Axio Scope A1, Carl Zeiss Co, Ltd). A total of 4 randomly-selected fields from at least 3 different tissue sections were selected for positive staining quantification. The mean optical density defined as the ratio of integrated optical density (IOD) to the corresponding cavity area was calculated using Image-Pro Plus software.

In order to further investigate angiogenesis, the analysis of microvessel density (MVD) based on CD31-positive staining was performed, as previously described (15). Slides were examined (magnification, x200) to identify the highest neovascularization area (hot spot) and a count of individual microvessels was conducted (magnification, $x 400$ ). The brown-staining single endothelial cells or cell clusters that visibly separated from adjacent microvessels were defined as a countable microvessels. The MVD was calculated in five representative areas of each slide. The evaluation was performed in a blinded manner by three independent investigators.

Terminal deoxynucleotidyl-transferase-mediated dUTP nick end labeling (TUNEL) assay. TUNEL assay was performed on bone sections using an ApopTag Plus Peroxidase In Situ Apoptosis kit (cat. no. S7101; EMD Millipore, Billerica, MA, USA) according to the manufacturer's protocol. Sections were deparaffinized, pretreated with proteinase $\mathrm{K}(20 \mu \mathrm{g} / \mathrm{ml}$; cat. no. 21627; EMD Millipore) and endogenous peroxidase was quenched using $\mathrm{H}_{2} \mathrm{O}_{2}(3 \%)$. Bone sections were incubated with a TdT enzyme (part\#90418 from ApopTag Plus Peroxidase In Situ Apoptosis kit; EMD Millipore), visualized with DAB detection system (5 min at room temperature) and counterstained in $0.5 \%(\mathrm{w} / \mathrm{v})$ methyl green $(10 \mathrm{~min}$ at room temperature). Stained sections were visualized under a light microscope (Zeiss Axio Scope A1, Carl Zeiss Co., Ltd.). Four randomly-selected fields from at least 3 different tissue sections were selected for positive staining quantification and the apoptosis rate (number of TUNEL-positive cells/number of all cells) was calculated in each section in a blinded manner by three independent investigators.

Statistical analysis. All data are presented as the mean \pm standard deviation. Following the verification of normality of distributions and equal variance, comparisons between groups were performed by Student's t-test. Statistics were performed using SPSS software (version 13.0; SPSS Inc., Chicago, IL, USA). $\mathrm{P}<0.05$ was considered to indicate a statistically significant difference.

\section{Results}

EPO treatment improves the microstructure of the femoral head in $\mathrm{ONFH}$. The micro-CT analysis from a 3-dimensional reconstruction demonstrated that bone trabeculae of femoral heads were more finely spread and intact in the EPO group compared with the control group (Fig. 1A-J). Trabeculae gradually lost their regular structure and connectivity in the control group. On the contrary, subchondral trabeculae in the EPO group maintained their normal structure and gradually recovered at 4,6 and 8 weeks. The quantitative analysis of micro-CT parameters, including BV/TV and BMD, confirmed that the microstructure of subchondral trabeculae in femoral heads from the EPO group were improved compared with the control group following 6 and 8 weeks of treatment (Fig. 1K and L). In particular, the BV/TV ratio and BMD of femoral heads at 6 and 8 weeks demonstrated significant differences between the two groups of interest $(\mathrm{P}<0.05$; Fig. $1 \mathrm{~K}$ and $\mathrm{L})$. However, $\mathrm{BV} / \mathrm{TV}$ and BMD did not differ significantly at 2 and 4 weeks following the EPO or saline treatment. These results suggest that prolonged administration of EPO can prevent bone loss of the femoral head in ONFH.

EPO treatment reduces the rate of empty lacunae and improves the trabecular bone volume in ONFH. As presented in Fig. 2, histological analysis of the H\&E staining revealed severely thinned and disordered trabeculae, large empty bone lacunae and numerous pyknotic nuclei of osteocytes in the femoral heads from the control group, which indicated that an animal model of ONFH was successfully established (Fig. 2A, C, E and G). In contrast, the histological analysis of femoral heads from the EPO group demonstrated that the microstructure of trabeculae gradually recovered (Fig. 2B, D, F and H). Consistent with the findings of the micro-CT assay, histological analyses of femoral heads demonstrated that trabecular bone volume displayed an increasing trend in mice in the EPO treatment group. Statistical analyses were performed between groups at multiple time points. BV/TV at 6 and 8 weeks in the EPO group was increased compared with the control group at the respective time points $(\mathrm{P}<0.05$; Fig. $2 \mathrm{I})$. In addition, in the EPO group, BV/TV at 8 weeks was increased compared with at 2 weeks $(\mathrm{P}<0.05$; Fig. 2I). Moreover, the proportion of empty bone lacunae at 2 and 4 weeks in the control group was higher than in the EPO group at corresponding time points $(\mathrm{P}<0.05$; Fig. 2J). These data are consistent with the hypothesis that administration of EPO can prevent bone loss in ONFH.

EPO treatment promotes expression of Runx2, osteocalcin, $V E G F$ and CD31 in ONFH. To further investigate the effect 

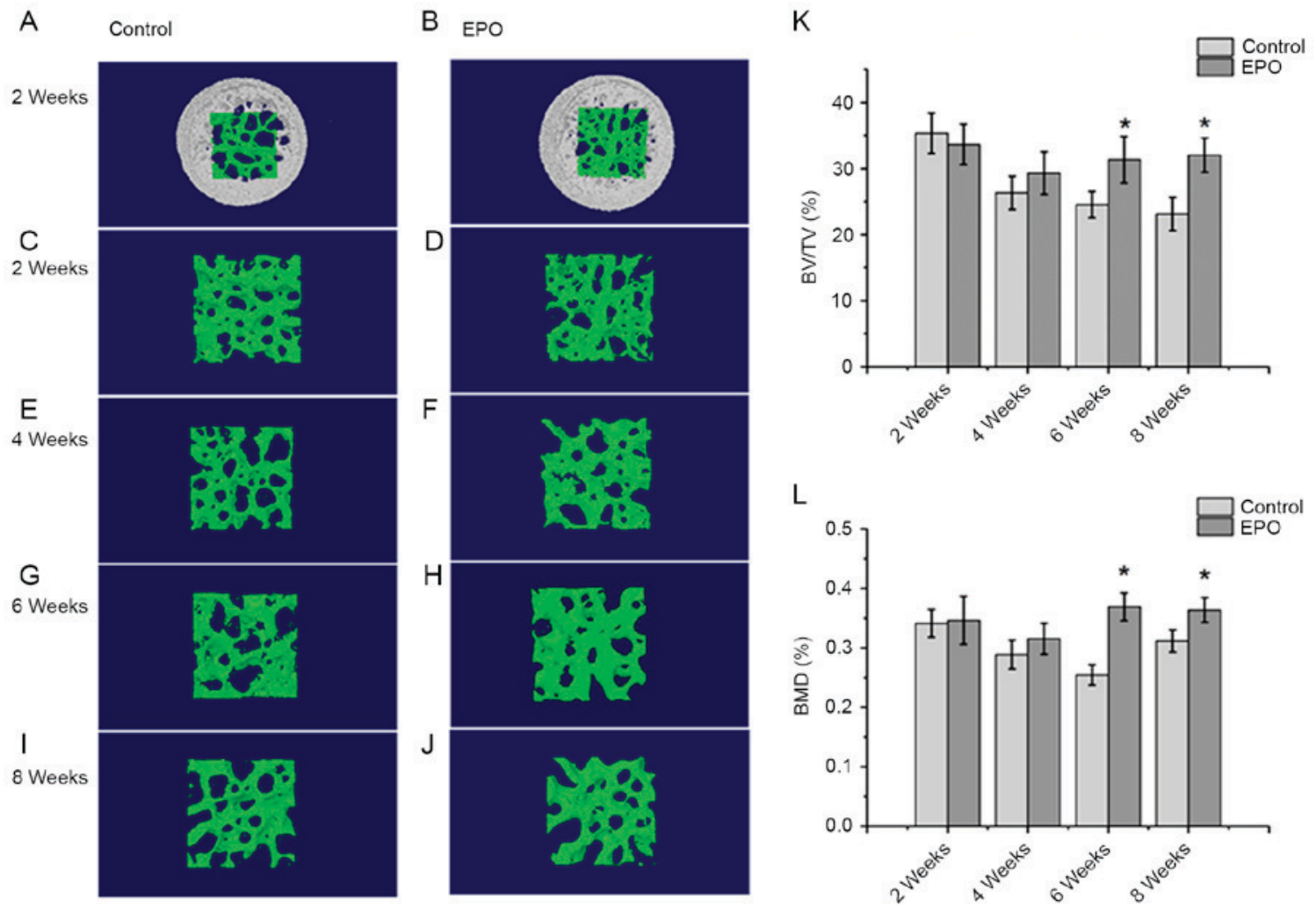

Figure 1. Micro-CT analysis of the trabeculae of femoral heads. At 2 weeks, the (A) control, (B) EPO, (C) control and (D) EPO groups. At 4 weeks, the (E) control and (F) EPO groups. At 6 weeks, the (G) control and (H) EPO groups. At 8 weeks, the (I) control and (J) EPO groups. The trabecular bone in the EPO treatment group recovered gradually compared with the control group. (K) Quantitative analysis of the bone volume; and (L) bone mineral density in the control and EPO treatment group at 2, 4, 6 and 8 weeks. The BV/TV and BMD of femoral heads increased in the EPO treatment group at 6 and 8 weeks compared with the control group. Data are presented as the mean \pm standard deviation. ${ }^{*} \mathrm{P}<0.05$ vs. the control group. EPO, erythropoietin; BV/TV, bone volume/total volume; BMD, bone mineral density; micro-CT, micro computed tomography.

of EPO on ONFH at 4 and 8 weeks following treatment with EPO, Runx2, osteocalcin, VEGF and CD31 were examined in the femoral heads by immunohistochemistry. The authors of the present study identified that positive signals for Runx2 and osteocalcin were significantly higher in the EPO group than in the control group $(\mathrm{P}<0.05$; Fig. 3$)$. In addition, an increased abundance of VEGF-positive staining was observed predominantly in the bone marrow cavity of femoral heads from the EPO group compared with the control group $(\mathrm{P}<0.05$; Fig. 4A-E) and the abundance of CD31-positive staining was increased in subchondral trabecular bone of necrotic femoral heads in the EPO group compared with the control group (Fig. 4F-I). The analysis of MVD, based on CD31-positive staining, demonstrated that few microvessels were present in subchondral trabecular bones of necrotic femoral heads in the control group at 4 and 8 weeks, while the number of microvessels in the EPO group increased significantly $(\mathrm{P}<0.05$; Fig. 4J). These data suggest that EPO can prevent bone loss in ONFH through enhancing Runx2-mediated osteogenesis and VEGF-mediated angiogenesis.

EPO treatment inhibits apoptosis induced by $O N F H$. At 4 and 8 weeks following treatment with EPO, TUNEL assays were performed to verify whether administration of EPO affected apoptosis associated with ONFH. It was determined that the treatment with EPO led to decreased abundance of apoptotic cells, both in the trabecular bone and bone marrow cavity at the same time points (Fig. 5A-D). In addition, quantitative analysis confirmed a significantly increased apoptosis rate in the control group compared with the EPO group at 4 and 8 weeks $(\mathrm{P}<0.05$; Fig. $5 \mathrm{E})$. These results suggest that the administration of EPO can inhibit apoptosis in the trabecular bone and bone marrow cavity in ONFH.

\section{Discussion}

ONFH is a multifactorial disease and a leading cause of mobility impairment. Long-term administration of GCs is considered one of the predominant pathological factors stimulating ONFH development and progression, leading to disruption of femoral head blood supply in the majority of cases. Resulting insufficient blood supply further affects the femoral head and eventually leads to its collapse and the development of hip osteoarthritis in patients (16). At present, there are no effective treatments for ONFH and the current therapy focuses on the management of symptoms. Hip replacement is the only effective treatment of choice. Therefore, there is a need for alternative interventions to treat ONFH and reduce patient morbidity and economic impact.

In previous studies, angiogenic and growth factors demonstrated promising effects on bone formation and regeneration in ONFH, including VEGF, fibroblast growth factors and hepatocyte growth factor (17-19). Several angiogenic factors could in theory be used for clinical treatment, but they could 
A

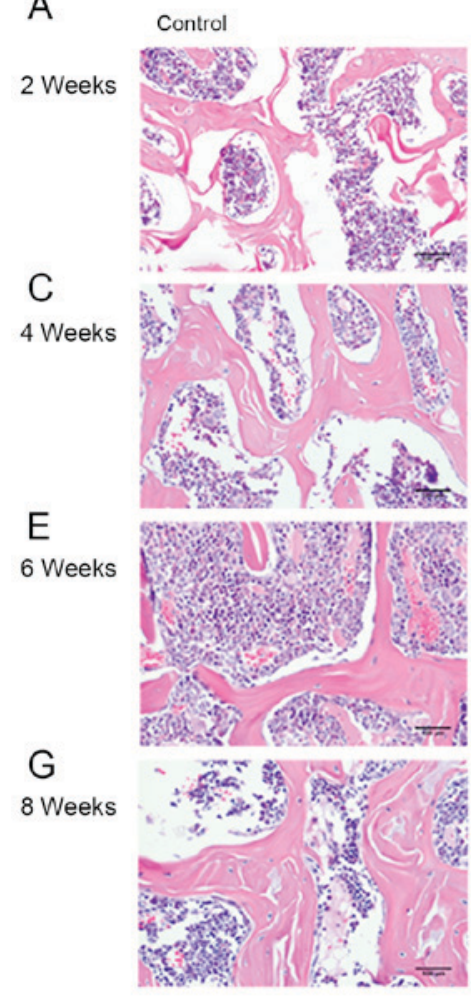

$B_{\text {EPO }}$

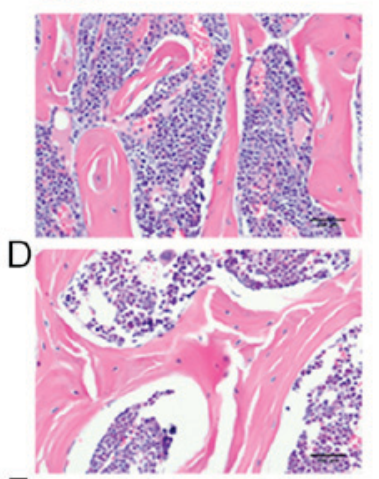

$\mathrm{F}$,

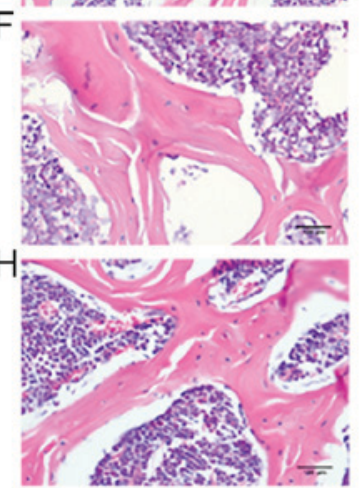

I $\square^{40}$ Genol

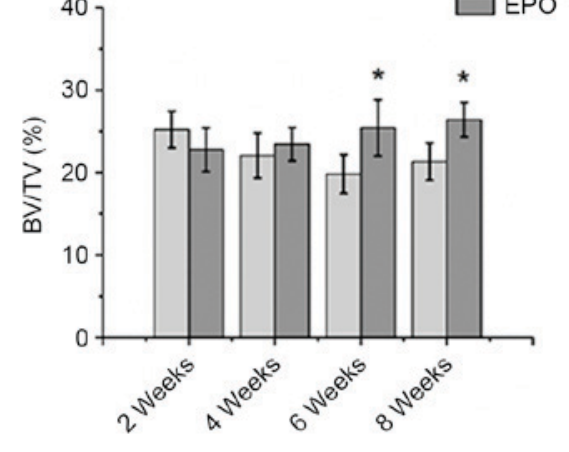

J 40 घ

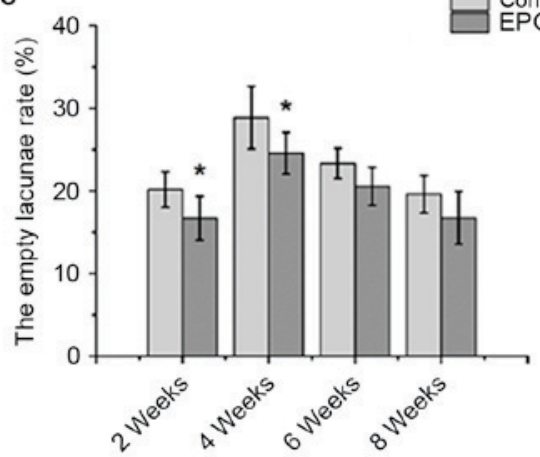

Figure 2. Histopathological analysis of the trabecular bone in the femoral head. At 2 weeks, the (A) control, (B) EPO, (C) control and (D) EPO groups. At 4 weeks, the (E) control and (F) EPO groups. At 6 weeks, the (G) control and (H) EPO groups. At 8 weeks, the (I) control and (J) EPO groups. Severely thinned and disordered trabeculae, large empty bone lacunae and numerous pyknotic nuclei of osteocytes were evident in femoral heads from the control group, whereas the morphological structure of EPO treatment group relatively improved (magnification, x400). (I) Quantitative analysis of bone volume; and (J) empty lacunae rate in the control and EPO treatment group at 2, 4, 6 and 8 weeks. The BV/TV of femoral heads increased in the EPO treatment group at 6 and 8 weeks compared with the control group. The empty lacunae rate in femoral heads decreased in the EPO treatment group at 2 and 4 weeks compared with the control group. Data are presented as the mean \pm standard deviation. ${ }^{*} \mathrm{P}<0.05$ vs. the control group. EPO, erythropoietin; BV/TV, bone volume/total volume.

also induce severe complications, including an increased intraosseous pressure due to vascular permeability, and arterial obliteration due to vascular smooth muscle proliferation (19). EPO is one of the most suitable candidates for the treatment of ONFH since it promotes bone regeneration $(3,4)$ and has been clinically used for the treatment of chronic anemia with few side effects (20). However, it has been demonstrated that systemic administration of EPO can deteriorate blood supply and blood viscosity as it increases RBC mass, and induces hypertension and thromboembolic events (21). In addition, a previous study reported that administration of 5,000 U/kg/day EPO can significantly increase the hematocrit, which can aggravate ONFH through impaired blood flow and nutrition and perfusion (22). In order to avoid these complications, the authors of the present study decided to intramuscularly administer $500 \mathrm{U} / \mathrm{kg} /$ day EPO. During the experimental period, no systemic side effects were observed.

Results from Micro-CT and histological analyses clearly demonstrated that administration of EPO restored microstructure of the femoral head by improving the trabecular bone volume and reducing the rate of empty lacunae in $\mathrm{ONFH}$. These observations are consistent with other studies which demonstrated that EPO treatment can enhance bone regeneration (5). Therefore, EPO treatment can improve ONFH in mice.

In order to further elucidate the mechanism underlying EPO-induced stimulation of bone formation in vivo, immunohistochemical analysis of Runx 2 and osteocalcin was performed. Runx 2 is a key transcription factor associated with osteoblast differentiation, and osteocalcin is a biochemical marker secreted by mature osteoblasts (23). Immunohistochemistry of femoral heads indicated that EPO treatment up-regulated the expression of Runx 2 and osteocalcin at 4 and 8 weeks. These results suggest that EPO may stimulate bone formation through Runx2-mediated osteogenesis. Other beneficial effects of EPO treatment include increased VEGF-associated angiogenesis (4). As a significant component of skeletal development and repair, angiogenesis contributes to the process of bone development and repair through adequate formation of new capillaries from existing vessels (24). In addition, GCs inhibit the expression of VEGF in femoral heads and consequently limit angiogenesis and osteogenesis in ONFH induced by GCs (7). Experiments in vitro demonstrated that primary osteoblasts derived from clinically osteoarthritic femoral heads displayed downregulation of VEGF following co-incubation with GCs for $24 \mathrm{~h}$ (25). In the present study, immunohistochemical analysis of VEGF demonstrated that the expression VEGF can be upregulated in the femoral heads in ONFH following treatment with EPO, compared with the control group. CD31, a member of the Ig superfamily of cell adhesion molecules, is commonly used to indicate the presence of endothelial cells (26). An analysis of MVD was performed based on CD31-positive staining 
A

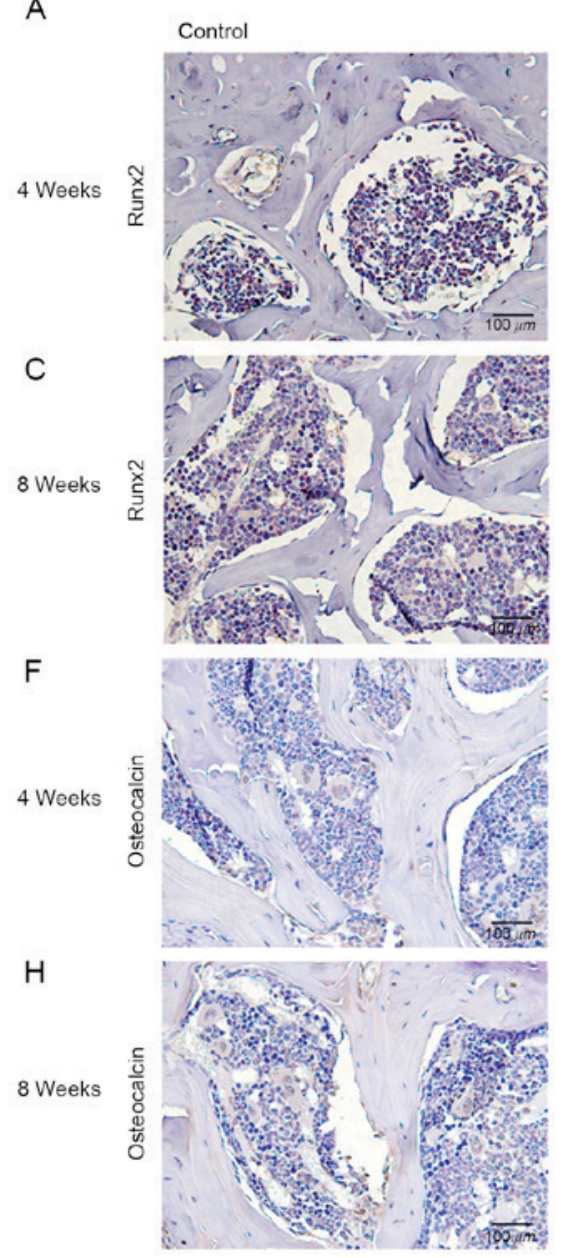

B EPO
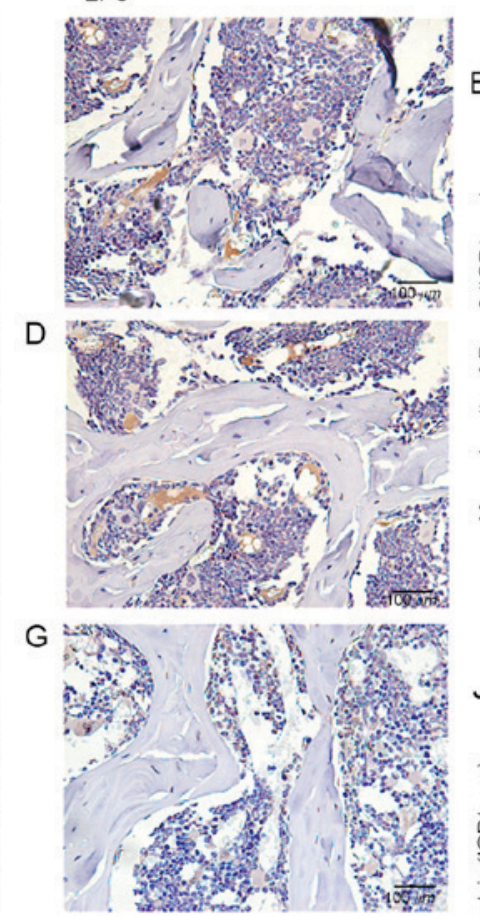

I

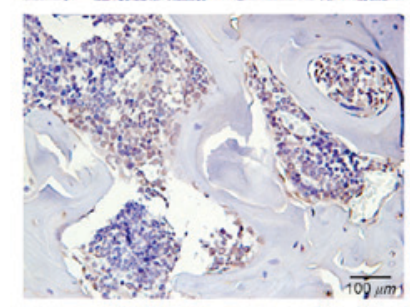

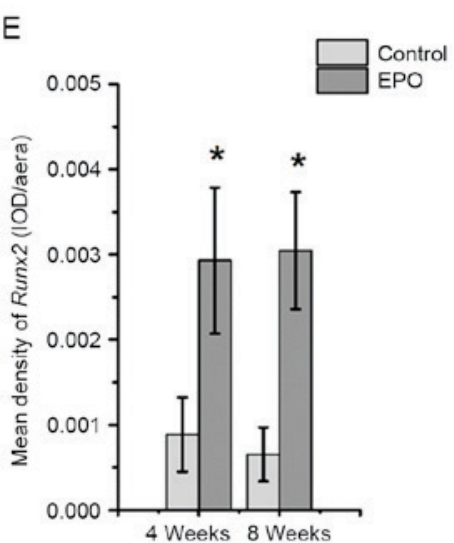

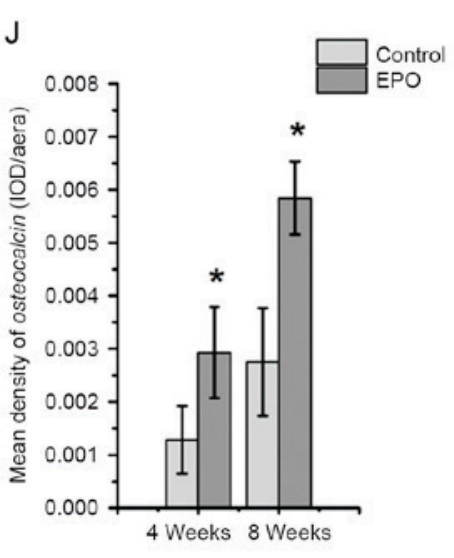

Figure 3. Immunohistochemical staining of Runx2 and osteocalcin. For Runx2, the (A) control and (B) EPO groups at 4 weeks, and the (C) control and (D) EPO groups at 8 weeks. (E) Quantification of Runx2 expression. For osteocalcin, the (F) control and (G) EPO groups at 4 weeks, and the (H) control and (I) EPO groups at 8 weeks. (J) Quantification of osteocalcin expression levels. Data are presented as the mean \pm standard deviation. ${ }^{*} \mathrm{P}<0.05$ vs. the control group. EPO, erythropoietin; Runx2, runt-related transcription factor 2; OD, optical density.

in femoral heads and it was demonstrated that microvessels were increased in the subchondral trabecular bone of necrotic femoral heads following treatment with EPO for 4 and 8 weeks, compared with the control group. Taken together, administration of EPO prevented bone loss in ONFH partially by increasing VEGF-mediated angiogenesis. It has been reported that VEGF is important for bone remodeling and regeneration as it effectively couples angiogenesis and osteogenesis in the bone microenvironment (27), but whether EPO-induced osteogenesis is coupled with VEGF remains to be elucidated.

Apoptosis of osteoblasts and osteocytes is a common pathogenic pathway of GC-induced ONFH (28). A previous study suggested that apoptosis partially causes cell death in the femoral head of patients with ONFH (29). In addition, O'Brien et al (30) reported that GCs could directly induce apoptosis of osteoblasts and osteocytes. Moreover, once the blood supply from collateral circulation is restricted without enough capillary arterialization in GC-induced $\mathrm{ONFH}$, the lack of available oxygen and nutrients may cause apoptosis of osteoblasts and osteocytes (31). Results of the TUNEL assay performed in the present study demonstrated that apoptosis occurred in osteocytes and bone marrow cells in the mice model of ONFH, while the apoptosis index in the EPO treatment group was significantly reduced at 4 and 8 weeks, suggesting that EPO could interfere with apoptosis and prevent bone loss in steroid-associated ONFH.

In conclusion, the present study demonstrated that the administration of EPO prevents bone loss by up-regulation of Runx2-mediated osteogenesis, an increase in VEGF-mediated angiogenesis and inhibition of cell apoptosis in the ONFH mouse model. Therefore, EPO is a promising agent for the treatment of ONFH. Further research will elucidate the mechanism underlying the effect of EPO treatment on the prevention of bone loss during ONFH while avoiding induction of side effects.

\section{Acknowledgements}

The present study was supported by the National Natural Science Foundation of China (grants nos. 81273770 and 81573994), the Natural Science Foundation of Zhejiang Province (grant no. LY16H270010), Traditional Chinese Medical Administration of Zhejiang Province (grant no. 2016ZA048) and the Cultivation Program for Innovative Talent Graduate Students (grant no. 311100G00901). The present study was also supported by the Program for Zhejiang 
A

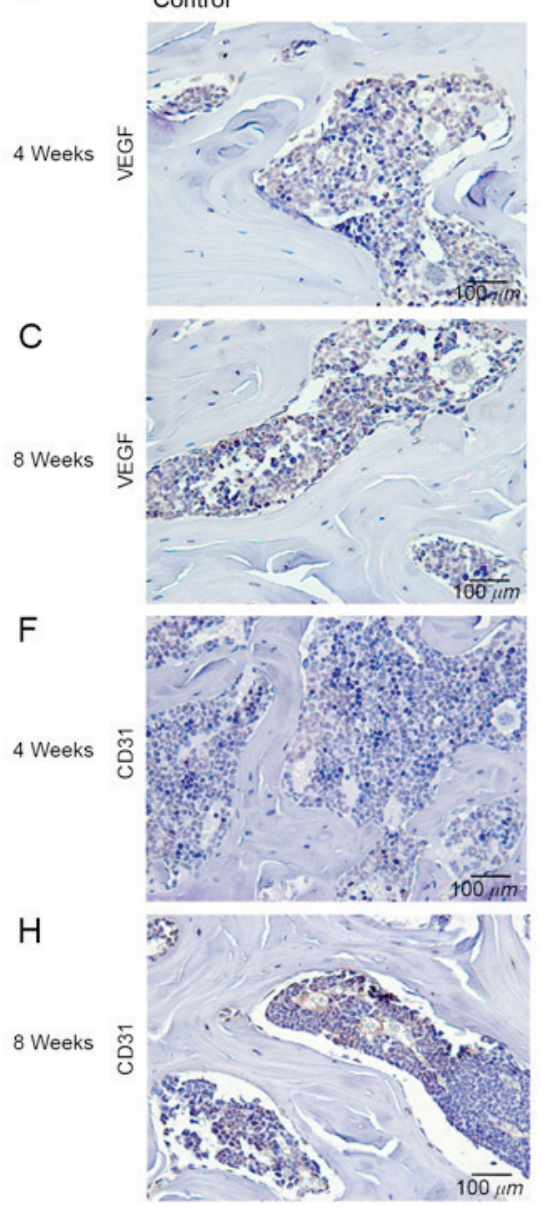

B EPO
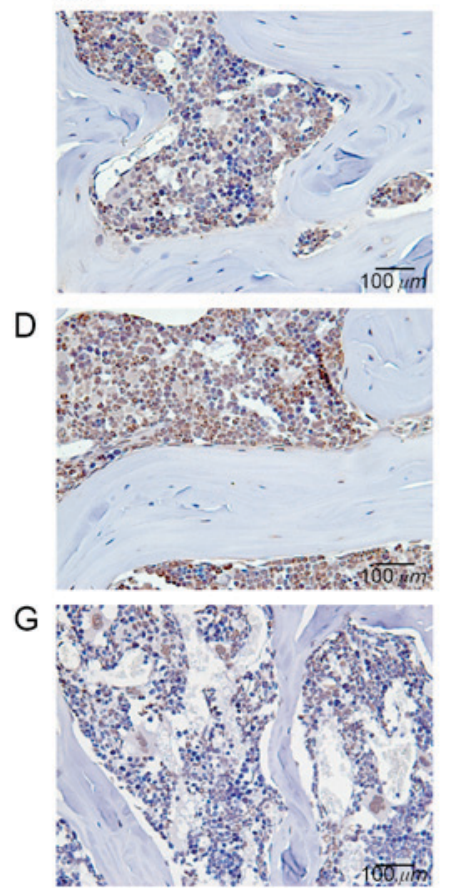

|

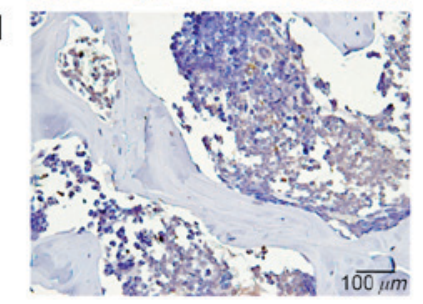

E
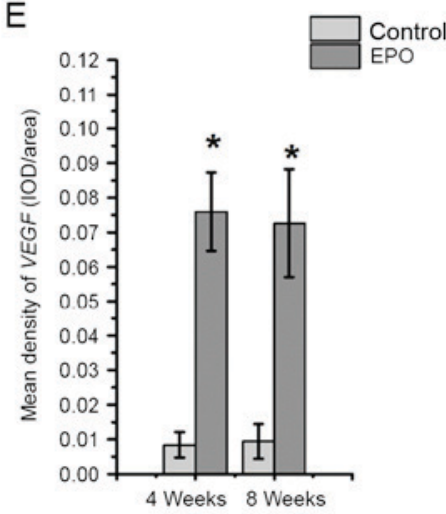

$J$

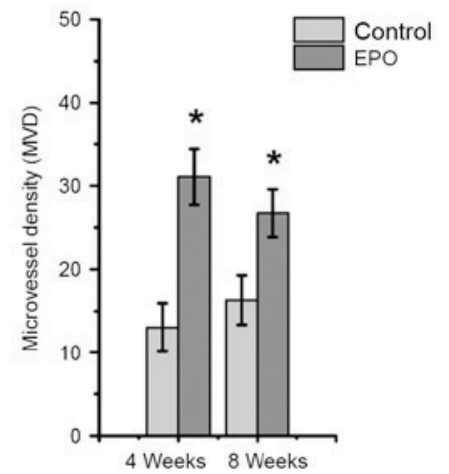

Figure 4. Immunohistochemical staining of VEGF and CD31 in of the femoral heads. For VEGF, the (A) control and (B) EPO groups at 4 weeks, and the (C) control and (D) EPO groups at 8 weeks. (E) Quantification of VEGF expression. For CD31, the (F) control and (G) EPO groups at 4 weeks, and the (H) control and (I) EPO groups at 8 weeks. (J) Quantification of CD31 expression levels. The mean density of VEGF in the EPO treatment group was higher than that in the control group at 4 and 8 weeks. MVD in the EPO treatment group significantly increased compared with the control group at 4 and 8 weeks. Data are presented as the mean \pm standard deviation. " $\mathrm{P}<0.05$ vs. the control group. VEGF, vascular endothelial growth factor; CD31, platelet endothelial cell adhesion molecule; EPO, erythropoietin; MVD, microvessel density.

A

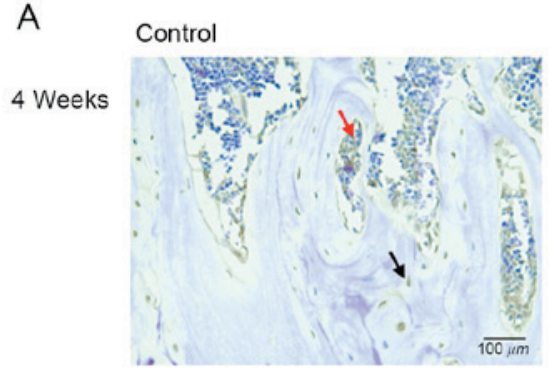

C 8 Weeks

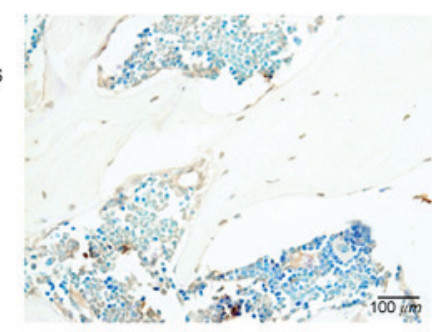

B

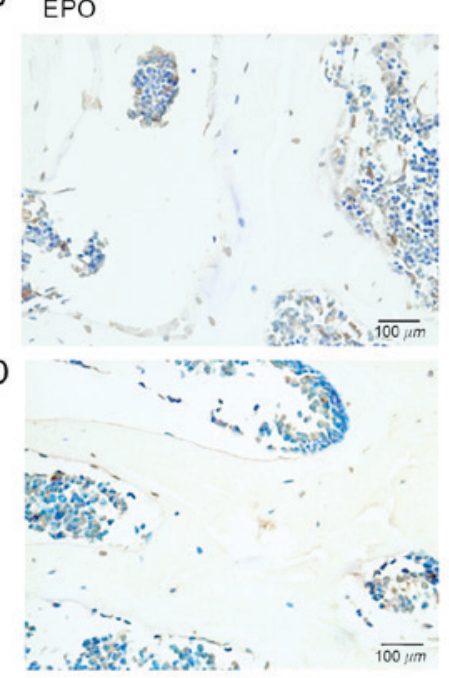

$\mathrm{E}$

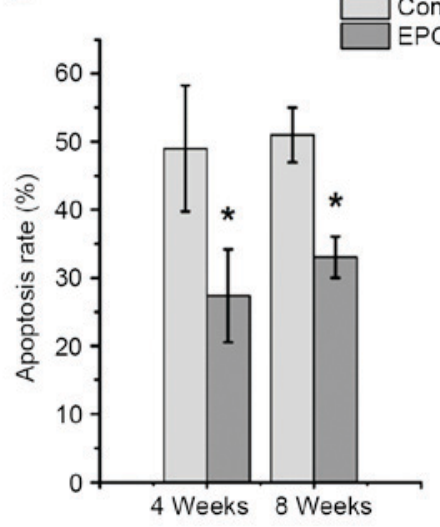

Figure 5. Terminal deoxynucleotidyl-transferase-mediated dUTP nick end labeling assays of femoral heads. The (A) control and (B) EPO groups at 4 weeks; the (C) control and (D) EPO groups at 8 weeks. At 4 and 8 weeks following treatment with EPO, less apoptotic cells were present in the trabecular bone and bone marrow cavity of the EPO group compared with the control group. The black arrow indicates an apoptotic cell in the trabecular bone and the red arrow indicates apoptotic cells in the bone marrow cavity. (E) Quantitative analysis of the apoptosis rate in the control and EPO treatment group at 4 and 8 weeks. A significantly higher apoptosis rate was observed in the control group compared with the EPO treatment group at 4 and 8 weeks. Data were presented as the mean \pm standard deviation. " $\mathrm{P}<0.05$ vs. the control group. EPO, erythropoietin. 
Leading Team of S\&T Innovation and Key Laboratory of Zhejiang Province.

\section{References}

1. Erlebacher A, Filvaroff EH, Gitelman SE and Derynck R: Toward a molecular understanding of skeletal development. Cell 80: 371-378, 1995.

2. Lombardero M, Kovacs K and Scheithauer BW: Erythropoietin: A hormone with multiple functions. Pathobiology 78: 41-53, 2011.

3. Hankenson KD, Dishowitz M, Gray C and Schenker M Angiogenesis in bone regeneration. Injury 42: 556-561, 2011.

4. Holstein JH, Orth M, Scheuer C, Tami A, Becker SC, Garcia P, Histing T, Mörsdorf P, Klein M, Pohlemann T and Menger MD: Erythropoietin stimulates bone formation, cell proliferation, and angiogenesis in a femoral segmental defect model in mice. Bone 49: 1037-1045, 2011.

5. Bakhshi H, Rasouli MR and Parvizi J: Can local Erythropoietin administration enhance bone regeneration in osteonecrosis of femoral head? Med Hypotheses 79: 154-156, 2012.

6. Childs SG: Osteonecrosis: Death of bone cells. Orthop Nurs 24: 295-303, 2005.

7. Weinstein RS, Wan C, Liu Q, Wang Y, Almeida M, O'Brien CA, Thostenson J, Roberson PK, Boskey AL, Clemens TL and Manolagas SC: Endogenous glucocorticoids decrease skeletal angiogenesis, vascularity, hydration, and strength in aged mice. Aging Cell 9: 147-161, 2010.

8. Uslu M, Kaya E, Yaykasli KO, Oktay M, Inanmaz ME, Işık C, Erdem H, Erkan ME and Kandiş H: Erythropoietin stimulates patellar tendon healing in rats. Knee 22: 461-468, 2015.

9. Zhang C, Zou YL, Ma J, Dang XQ and Wang KZ: Apoptosis associated with Wnt/ $\beta$-catenin pathway leads to steroid-induced avascular necrosis of femoral head. BMC Musculoskelet Disord 16: 132, 2015.

10. Weinstein RS, Nicholas RW and Manolagas SC: Apoptosis of osteocytes in glucocorticoid-induced osteonecrosis of the hip. J Clin Endocrinol Metab 85: 2907-2912, 2000.

11. Ryoo S, Lee S, Jo S, Lee S, Kwak A, Kim E, Lee J, Hong J, Jhun H, Lee Y, et al: Effect of lipopolysaccharide (LPS) on mouse model of steroid-induced avascular necrosis in the femoral head (ANFH). J Microbiol Biotechnol 24: 394-400, 2014

12. Bouxsein ML, Boyd SK, Christiansen BA, Guldberg RE, Jepsen KJ and Müller R: Guidelines for assessment of bone microstructure in rodents using micro-computed tomography. J Bone Miner Res 25: 1468-1486, 2010.

13. Qin L, Zhang G, Sheng H, Yeung KW, Yeung HY, Chan CW, Cheung WH, Griffith J, Chiu KH and Leung KS: Multiple bioimaging modalities in evaluation of an experimental osteonecrosis induced by a combination of lipopolysaccharide and methylprednisolone. Bone 39: 863-871, 2006.

14. Okazaki S, Nishitani Y, Nagoya S, Kaya M, Yamashita T and Matsumoto H: Femoral head osteonecrosis can be caused by disruption of the systemic immune response via the toll-like receptor 4 signalling pathway. Rheumatology (Oxford) 48: 227-232, 2009 .
15. Weidner N, Semple JP, Welch WR and Folkman J: Tumor angiogenesis and metastasis-correlation in invasive breast carcinoma. N Engl J Med 324: 1-8, 1991.

16. Babis GC, Sakellariou V, Parvizi J and Soucacos P: Osteonecrosis of the femoral head. Orthopedics 34: 39, 2011.

17. Young S, Patel ZS, Kretlow JD, Murphy MB, Mountziaris PM, Baggett LS, Ueda H, Tabata Y, Jansen JA, Wong M and Mikos AG: Dose effect of dual delivery of vascular endothelial growth factor and bone morphogenetic protein-2 on bone regeneration in a rat critical-size defect model. Tissue Eng Part A 15: 2347-2362, 2009

18. Kuroda Y, Akiyama H, Kawanabe K, Tabata Y and Nakamura T: Treatment of experimental osteonecrosis of the hip in adult rabbits with a single local injection of recombinant human FGF-2 microspheres. J Bone Miner Metab 28: 608-616, 2010.

19. Wen Q, Ma L, Chen YP, Yang L, Luo W and Wang XN: Treatment of avascular necrosis of the femoral head by hepatocyte growth factor-transgenic bone marrow stromal stem cells. Gene Ther 15: 1523-1535, 2008

20. Haroon ZA, Amin K, Jiang X and Arcasoy MO: A novel role for erythropoietin during fibrin-induced wound-healing response. Am J Pathol 163: 993-1000, 2003.

21. Maiese K, Chong ZZ and Shang YC: Raves and risks for erythropoietin. Cytokine Growth Factor Rev 19: 145-155, 2008.

22. Rezaeian F, Wettstein R, Amon M, Scheuer C, Schramm R, Menger MD, Pittet B and Harder Y: Erythropoietin protects critically perfused flap tissue. Ann Surg 248: 919-929, 2008.

23. Lee NK, Sowa H, Hinoi E, Ferron M, Ahn JD, Confavreux C, Dacquin R, Mee PJ, McKee MD, Jung DY, et al: Endocrine regulation of energy metabolism by the skeleton. Cell 130: 456-469, 2007.

24. Winet $\mathrm{H}$ : The role of microvasculature in normal and perturbed bone healing as revealed by intravital microscopy. Bone 19 (1 Suppl): 39S-57S, 1996.

25. Varoga D, Drescher W, Pufe M, Groth G and Pufe T: Differential expression of vascular endothelial growth factor in glucocorticoid-related osteonecrosis of the femoral head. Clin Orthop Relat Res 467: 3273-3282, 2009.

26. Sumpio BE, Yun S, Cordova AC, Haga M, Zhang J, Koh Y and Madri JA: MAPKs (ERK1/2, p38) and AKT can be phosphorylated by shear stress independently of platelet endothelial cell adhesion molecule-1 (CD31) in vascular endothelial cells. J Biol Chem 280: 11185-11191, 2005.

27. Clarkin CE and Gerstenfeld LC: VEGF and bone cell signalling: An essential vessel for communication? Cell Biochem Funct 31: 1-11, 2013.

28. Weinstein RS: Glucocorticoid-induced osteonecrosis Endocrine 41: 183-190, 2012.

29. Calder JD, Buttery L, Revell PA, Pearse M and Polak JM: Apoptosis-a significant cause of bone cell death in osteonecrosis of the femoral head. J Bone Joint Surg Br 86: 1209-1213, 2004.

30. O'Brien CA, Jia D, Plotkin LI, Bellido T, Powers CC, Stewart SA, Manolagas SC and Weinstein RS: Glucocorticoids act directly on osteoblasts and osteocytes to induce their apoptosis and reduce bone formation and strength. Endocrinology 145: 1835-1841, 2004

31. Kaushik AP, Das A and Cui Q: Osteonecrosis of the femoral head: An update in year 2012. World J Orthop 3: 49-57, 2012. 